\title{
Constraints on submicrojansky radio number counts based on evolving VLA-COSMOS luminosity functions
}

\author{
M. Novak ${ }^{1,2}$, V. Smolčić ${ }^{1}$, E. Schinnerer ${ }^{2}$, G. Zamorani ${ }^{3}$, I. Delvecchio ${ }^{1}$, M. Bondii ${ }^{4}$, and J. Delhaize ${ }^{1}$ \\ ${ }^{1}$ Department of Physics, Faculty of Science, University of Zagreb, Bijenička cesta 32, 10000 Zagreb, Croatia \\ e-mail: novak@mpia-hd.mpg.de \\ 2 Max-Planck-Institut für Astronomie, Königstuhl 17, 69117 Heidelberg, Germany \\ ${ }^{3}$ INAF - Osservatorio Astronomico di Bologna, Via Piero Gobetti 93/3, 40129 Bologna, Italy \\ ${ }^{4}$ INAF - Istituto di Radioastronomia di Bologna, Via Piero Gobetti 101, 40129 Bologna, Italy
}

Received 24 July 2017 / Accepted 23 February 2018

\begin{abstract}
We present an investigation of radio luminosity functions (LFs) and number counts based on the Karl G. Jansky Very Large ArrayCOSMOS $3 \mathrm{GHz}$ Large Project. The radio-selected sample of 7826 galaxies with robust optical/near-infrared counterparts with excellent photometric coverage allows us to construct the total radio LF since $z \sim 5.7$. Using the Markov chain Monte Carlo algorithm, we fit the redshift dependent pure luminosity evolution model to the data and compare it with previously published VLA-COSMOS LFs obtained on individual populations of radio-selected star-forming galaxies and galaxies hosting active galactic nuclei classified on the basis of presence or absence of a radio excess with respect to the star-formation rates derived from the infrared emission. We find they are in excellent agreement, thus showing the reliability of the radio excess method in selecting these two galaxy populations at radio wavelengths. We study radio number counts down to submicrojansky levels drawn from different models of evolving LFs. We show that our evolving LFs are able to reproduce the observed radio sky brightness, even though we rely on extrapolations toward the faint end. Our results also imply that no new radio-emitting galaxy population is present below $1 \mu \mathrm{Jy}$. Our work suggests that selecting galaxies with radio flux densities between 0.1 and $10 \mu \mathrm{Jy}$ will yield a star-forming galaxy in $90-95 \%$ of the cases with a high percentage of these galaxies existing around a redshift of $z \sim 2$, thus providing useful constraints for planned surveys with the Square Kilometer Array and its precursors.
\end{abstract}

Key words. galaxies: evolution - radio continuum: galaxies

\section{Introduction}

Radio emission in galaxies below rest-frame frequencies of $30 \mathrm{GHz}$ is mostly synchrotron radiation arising from cosmic electrons gyrating in the galaxy magnetic fields, with a fraction of thermal emission due to free-free processes (e.g., Sadler et al. 1989; Condon 1992; Clemens et al. 2008; Tabatabaei et al. 2017). Based on the source of acceleration of these cosmic electrons, galaxies can be separated into two categories, as follows. The electrons are being accelerated by supernovae in star-forming (SF) galaxies, and by an accreting supermassive black hole $(\mathrm{SMBH})$ in active galactic nuclei (AGN). It is often not possible to make a clear distinction between these two types as many galaxies are composites.

Several classification schemes for radio AGN exist, but they are not fully consistent with each other as they are based either solely on properties observed in the radio or on a combination of host properties (e.g., Smolčić 2015). For example, one method uses the ratio of radio-to-optical flux densities to divide the AGN into radio-loud (RL) and radio-quiet (RQ) AGN (e.g., Bonzini et al. 2013; Padovani et al. 2015). A different method analyzes optical emission lines to classify AGN into low- and high-excitation galaxies (LEGs and HEGs, respectively, e.g., Laing et al. 1994; Hardcastle et al. 2006; Smolčić 2009). We use the latter nomenclature for our radio sources to keep the AGN classes as general as possible, noting that the presence of a radio detection is not necessary for such a division since it is based on the host properties (for a review of the extensive nomenclature of radio AGN, see also Padovani et al. 2017). These observational constraints are used to probe the underlying physical mechanism, which can be best understood through SMBH accretion efficiency, that is, the amount of gravitational energy converted into radiation (see Heckman \& Best 2014). An AGN with a luminosity higher than $1-10 \%$ of the Eddington limit can be considered radiatively efficient (this category largely overlaps with HEGs); it radiates energy mostly isotropically (e.g., Fanidakis et al. 2011; Heckman \& Best 2014). On the other hand, radiatively inefficient AGN (overlapping with LEGs) have luminosities below 1-10\% of the Eddington limit, and the energy is emitted mostly mechanically in the form of collimated jets (see also Evans et al. 2006; Merloni \& Heinz 2008). The difference between the two types of AGN can also be seen in the properties of their galaxy hosts and their environments. Typically, HEGs have lower stellar and SMBH masses, but higher gas masses. Compared to LEGs, they also have bluer (green) colors and reside almost exclusively in less dense environments (e.g., Best \& Heckman 2012; Gendre et al. 2013). LEGs are usually hosted by massive red galaxies residing in denser environments (see also Hale et al. 2018). These observations are consistent with the idea that SMBHs of HEGs are fueled by accretion of cold gas, which is possibly being enriched through galaxy mergers, while SMBHs of LEGs obtain their gas supply from the cluster halo (for more details, see Best \& Heckman 2012). It has also been shown that these two AGN types evolve differently with cosmic time. At lower luminosities, 
HEGs are less abundant, but evolve strongly (similar to SF galaxies), while LEGs show little or no evolution at all (e.g., Pracy et al. 2016).

Analysis of emission lines in the optical spectra has proven to be a very successful method for identifying different types of AGN and separating them from SF galaxies, but the origin of the radio emission in AGN selected in this way might not be necessarily linked to SMBH accretion processes or jets alone. Previous studies have shown that HEGs have higher dust temperatures, significant star formation rates, and obey the infrared (IR)-radio correlation valid for SF galaxies (e.g., Morić et al. 2010; Hardcastle et al. 2013). Delvecchio et al. (2017) find that only $30 \%$ of AGN classified via X-ray or mid-IR signatures show a significant radio excess compared to the total IR emission arising from star formation. In the remainder of the sample that were not classified as X-ray/IR AGN, they showed that some AGN appear as "red and dead" galaxies in all bands except the radio (see also Heckman \& Best 2014). When starting from a radio-selected sample, the division between the two AGN classes is less obvious because many galaxies are composites. The well-known radio loudness dichotomy emerges when radio emission is analyzed starting from an optically selected sample of AGN (e.g., Kimball et al. 2011; Baloković et al. 2012). For the purpose of using radio emission as a proxy for measuring star formation rates (SFRs) or AGN feedback, it is important to estimate which process dominates the radio emission: star formation processes, or SMBH accretion. For this reason, we consider many HEGs as SF galaxies even though they show a clear AGN contribution in non-radio bands. Their radio emission, which is investigated here, is consistent with originating from star formation processes in the host galaxy (see also Smolčić et al. 2017b for a more detailed discussion of this).

One way to constrain how different populations add up to the total radio sky is through source number counts, that is, the number of galaxies contained in a solid angle of the sky with a given flux density (for a review from a radio perspective, see, e.g., Padovani 2016). First used as a tool for studying the geometry of the Universe, source number counts became a practical method for tracing galaxy evolution. Such studies can also provide a statistical way to search for and describe new populations of galaxies (e.g., Longair 1966; Franzen et al. 2014). Flattening of Euclidean normalized number counts at submicrojansky flux densities at $1.4 \mathrm{GHz}$ indicated the surfacing of a new galaxy population in the radio (e.g., Rowan-Robinson et al. 1993; Seymour et al. 2004; Padovani et al. 2009), but the scatter in the observed counts from different datasets was significant (e.g., Hopkins et al. 2003; Heywood et al. 2013). There were also some indications that the $1.4 \mathrm{GHz}$ counts could remain flat down to $10 \mu \mathrm{Jy}$ or might even rise (e.g., Owen \& Morrison 2008). Fixsen et al. (2011) and Vernstrom et al. (2011) discussed in detail the implications of the observed radio excess from $\mathrm{ARCADE}^{1} 2$ sky brightness measurements, which suggested the existence of an abundant, but faint new radio population (possibly due to diffuse emission from clusters or halos or dark matter annihilation). However, recent deep VLA observations show evidence of a further steepening of the number counts below $\sim 50 \mu \mathrm{Jy}$ at $1.4 \mathrm{GHz}$ that is consistent with the idea of SF galaxies dominating the faint radio sky (e.g., Vernstrom et al. 2016a; Smolčić et al. 2017a).

\footnotetext{
1 Absolute Radiometer for Cosmology, Astrophysics and Diffuse Emission.
}

There are several methods applicable for calculating source number counts. The traditional method relies on counting discrete sources in flux density bins (see, e.g., de Zotti et al. 2010). It is the most straightforward approach, although subject to biases, such as non-uniform rms across the radio map, or resolution bias, and correct for such incompleteness issues (e.g., Hales et al. 2014; Vernstrom et al. 2016b; Smolčić et al. 2017a). Another approach is a blind probability of deflection analysis $P(D)$ performed on a confusion-limited survey (see Scheuer 1957). This was recently used on deep Karl G. Jansky Very Large Array (VLA) data by Condon et al. (2012) and Vernstrom et al. (2014). The method measures the bias in the noise and relies on a small number of assumptions. The advantage of the $P(D)$ analysis is that it can also model source counts well below the usual $5 \sigma$ sensitivity limit. However, because of its blind nature, it yields no information on specific sub-populations of galaxies. This method cannot be applied to high-resolution maps when the map is no longer dominated by confusion noise. In this case, one possible option is to stack the radio map at preset positions drawn from a catalog of priors derived through higher sensitivity (not necessarily radio) observations (for a Bayesian approach, see, e.g., Mitchell-Wynne et al. 2014; Zwart et al. 2015b). This method also allows probing the radio map below the nominal sensitivity threshold, but careful assessment of biases is required (see also Zwart et al. 2015a).

If a cosmological model for the Universe is assumed, the radio number counts are a natural consequence of the underlying luminosity functions (LFs; Condon 1989). Recently, Novak et al. (2017), hereafter N17, and Smolčić et al. (2017c), hereafter S17c, published LFs of SF galaxies and AGN, respectively, using the VLA-COSMOS $3 \mathrm{GHz}$ Large Project data (Smolčić et al. 2017a). In this work we use LFs to estimate the number counts, relative fractions of different galaxy sub-populations, and redshift distributions of SF galaxies down to submicrojansky flux density levels. Furthermore, we try to fit the total measured radio LF with different models of evolving LFs. Such a fit no longer relies on classifications of individual galaxies, but only on the analytical representation of the LF of the global population. This approach helps in mitigating biases introduced by misclassifications of galaxies. The deep COSMOS field is optimized to probe faint distant sources that will predominantly be SF galaxies. To successfully distinguish the evolutions of HEGs and LEGs from the radio perspective, wide and shallow surveys are additionally needed to probe the bright luminosity end where the two AGN populations change their relative fractions $\left(L_{1.4 \mathrm{GHz}} \gtrsim 10^{26} \mathrm{~W} \mathrm{~Hz}^{-1}\right.$, e.g., Pracy et al. 2016). Constraints on the faint radio populations are useful for future radio surveys such as the $\mathrm{SKA}^{2}, \mathrm{ASKAP}^{3}, \mathrm{EMU}^{4}$ and MeerKAT ${ }^{5}$ (see also Norris et al. 2013). In this paper we show that future radio observations will provide an additional valuable and simple galaxy classification method based on the observed radio flux density alone.

In Sect. 2 we briefly describe the radio and the ancillary data used throughout this work. In Sect. 3 we focus on methods for deriving the radio LF and its evolution through cosmic time. We also discuss biases that might affect our results. In Sect. 4 we discuss the radio number counts derived from radio LFs and implications for future radio surveys. Finally, in Sect. 5 we provide a brief summary.

\footnotetext{
Square Kilometer Array.

Australian SKA Pathfinder.

4 Evolutionary Map of the Universe.

Meer Karoo Array Telescope.
} 
We assume flat concordance lambda cold dark matter $(\Lambda \mathrm{CDM})$ cosmology defined with a Hubble constant of $H_{0}=70 \mathrm{~km} \mathrm{~s}^{-1} \mathrm{Mpc}^{-1}$, dark energy density of $\Omega_{\Lambda}=0.7$, and matter density of $\Omega_{\mathrm{m}}=0.3$. For the radio spectral energy distribution we assume a simple power law described as $S_{v} \propto v^{\alpha}$, where $S_{v}$ is the flux density at frequency $v$ and $\alpha$ is the spectral index. If not explicitly stated otherwise, $\alpha=-0.7$ is assumed.

\section{Data}

The data used throughout the paper are radio selected and have been cross-correlated with the multiwavelength optical/nearinfrared (NIR) and value-added catalogs. The radio data were taken with the VLA at $3 \mathrm{GHz}$, reaching a sensitivity of $2.3 \mu \mathrm{Jy}$ beam $^{-1}$ at a resolution of 0.75 . The observational setup, data reduction, source extraction, and completeness simulations are described in detail in Smolčić et al. (2017a). In short, $384 \mathrm{hr}$ of observations were conducted with the VLA in $S$-band (2-4 GHz continuum) to uniformly cover the $2 \mathrm{deg}^{2}$ COSMOS field with 192 pointings. Both the VLA A and C configurations were used. The scatter of the flux calibration in the different pointings is $5 \%$ around the mean, ensuring good flux measurements. The data were imaged with the multiscale multifrequency synthesis (Rau \& Cornwell 2011) to obtain the intensity (Stokes I) radio map. The radio components above $5 \sigma$ were extracted using BLOBCAT (Hales et al. 2012), and were further inspected by eye where necessary. The final source catalog contains around 5000 galaxies per square degree. The completeness of the $3 \mathrm{GHz}$ catalog was estimated through Monte Carlo simulations of mock sources, and it was given as a function of flux density. The catalog is $60 \%$ complete below $20 \mu \mathrm{Jy}, 95 \%$ above $40 \mu \mathrm{Jy}$, and a $100 \%$ completeness is assumed above $100 \mu \mathrm{Jy}$.

Radio sources were assigned counterparts (for details see Smolčić et al. 2017b) primarily from the COSMOS2015 cata$\log$ (Laigle et al. 2016). The COSMOS2015 catalog contains the high-quality multiwavelength photometry of $\sim 800000$ sources across more than 30 bands from near-ultraviolet (NUV) to NIR obtained from GALEX, UltraVISTA DR2, Subaru/HyperSuprime-Cam, and the SPLASH Spitzer legacy program. Sources were identified in the $\chi^{2}$ weighted stack of $z^{++} Y J H K_{s}$ bands. The photometry extraction was performed with SEXTRACTOR within $2^{\prime \prime}$ and $3^{\prime \prime}$ apertures on individual bands that were previously homogenized to a common pointspread-function (resolution of $0 . ' 8$ ). For higher counterpart completeness, additional sources were considered from the $i$-band selected catalog (Capak et al. 2007) and the $3.6 \mu \mathrm{m}$ Spitzer/IRAC 6 catalog (Saunders et al. 1990).

The process of the cross-correlation of the $3 \mathrm{GHz}$ radio and multiwavelength sources is explained in detail in Smolčić et al. (2017b). Associations of radio sources to NIR/optical sources were based on the nearest-neighbor matching within a search radius of $0 .^{\prime} 8$ for the COSMOS2015/i-band, and a radius of 1.'7 for the IRAC catalog. Additionally, false-match probabilities were drawn from Monte Carlo simulations using a background model that mimics the observed $m_{3.6 \mu \mathrm{m}}$ magnitude distribution of radio counterparts. The entire process resulted in 8035 radio sources with optical/NIR counterparts across an area of $1.77 \mathrm{deg}^{2}$ free from saturation and bright stars' contamination (7729 from COSMOS2015, 97 from $i$-band and 209 from the IRAC catalogs). We do not use sources assigned an IRAC counterpart as robust photometric redshifts cannot be obtained from the IRAC data alone.

\footnotetext{
6 Infrared Array Camera.
}

A reliable photometric redshift estimate is available for 7826 radio sources associated with counterparts in the COSMOS2015 or the $i$-band selected catalog. These sources form our final radio selected sample used throughout. This final sample represents $90 \%$ of all cataloged radio sources. Of the remaining $10 \%$, one half of the sources have a low signal-tonoise ratio $(\mathrm{S} / \mathrm{N})$ of $<6$, making many of them likely candidates for spurious detections. It was estimated by Smolčić et al. (2017a) that $3 \%$ of the sources in the $3 \mathrm{GHz}$ radio catalog are expected to be spurious (mostly those with $S / N<5.5$ ). By taking into account the false-detection probability of the VLA-COSMOS $3 \mathrm{GHz}$ radio survey, we estimate that $7 \%$ of real radio sources were not assigned a counterpart (this includes IRAC associations), introducing a small negative bias into our counting statistics. It is likely that the redshift distribution of these unaccounted sources is not uniform across all redshifts, but skewed toward high redshift. This would imply somewhat higher densities of sources at high redshifts at a given luminosity than suggested by the data. In the absence of an actual redshift distribution of these sources, we do not attempt to correct for this bias.

Spectroscopic redshift is available for $35 \%$ of our sources, mostly below $z<1.5$. The median accuracy of the photometric redshifts in our sample is $\Delta z /\left(1+z_{\text {spec }}\right)=0.01(0.04)$, with a catastrophic failure rate of $4 \%(12 \%)$ for redshift range $z>0$ $(z>1.5)$.

\section{Luminosity functions and evolution}

We first describe the methods used to measure the total radio LFs from the VLA-COSMOS $3 \mathrm{GHz}$ data. Then, we briefly summarize the results from N17 and S17c, where the authors focused on individual galaxy populations: SF and AGN, respectively. Finally, we fit different evolution models of the total radio LF and discuss the various models as well as potential biases in the analysis.

\subsection{Measuring the total radio luminosity function}

To calculate the total radio LFs, we followed the procedure that was used in N17. The method is based on computing the maximum observable volume, $V_{\max }$, for each source (Schmidt 1968) and simultaneously applying completeness corrections that take into account the nonuniform $\mathrm{rms}$ noise and the resolution bias (see Sect. 3.1 in N17). We did not apply any statistical corrections for radio sources without assigned optical counterparts as the redshift distribution is unknown, but we expect these effects to be less than $10 \%$ (see also Sect. 2). In order to obtain the rest-frame luminosity of a radio source, a spectral index must be assumed. The spectral index of a source is calculated between 1.4 and $3 \mathrm{GHz}$, if a $1.4 \mathrm{GHz}$ detection (Schinnerer et al. 2010) is available, and assumed to remain unchanged at all radio frequencies. Such spectral indices are available for a quarter of the sample. For sources detected only at $3 \mathrm{GHz}$, we assumed $\alpha=-0.7$, which corresponds to the average spectral index of the entire $3 \mathrm{GHz}$ population (see Sect. 4 in Smolčić et al. 2017a). Redshift bins were chosen to be large enough not to be affected severely by photometric redshift uncertainty. Luminosity bins in each redshift bin span exactly the observed luminosity range of the data. The lowest luminosity bin spans from the faintest observed source to the $5 \sigma$ detection threshold at the upper redshift limit (corresponding to $5 \times 2.3 \mu \mathrm{Jy} \mathrm{beam}^{-1}$ at $3 \mathrm{GHz}$ ) so that possible issues due to poorer sampling can be mitigated more easily. The reported luminosity for each LF is the median luminosity of the sources within the bin. The horizontal bars in the 
plots correspond to the width of the bin. The total radio LFs are shown in Fig. 1 (black points) and are also listed in Table A.1. In an attempt to quantify the bias introduced by an imperfect radio $\mathrm{K}$ correction, which will mostly affect the bright end of the LF, the total radio LF was also calculated assuming a spectral index of $\alpha=-0.7$ for all sources (see green hexagons in Fig. 1). We discuss this further below.

\subsection{Star-forming galaxies and AGN}

$\mathrm{N} 17$ and S17c classified galaxies based on the radio emission excess compared to the IR based SFR, following the prescription presented in Delvecchio et al. (2017). This method was aimed at distributing galaxies into two categories depending on the physical process likely producing the radio emission. Galaxies that exhibit a $3 \sigma$ significant radio excess, namely

$\log \left(\frac{L_{1.4 \mathrm{GHz}}\left[\mathrm{W} \mathrm{Hz}^{-1}\right]}{\mathrm{SFR}_{\mathrm{IR}}\left[M_{\odot} \mathrm{yr}^{-1}\right]}\right)>a(1+z)^{b}$,

where $a=22.0$ and $b=0.013$, owe at least $80 \%$ of their radio emission to the AGN, while the rest is due to star formation. The percentage of AGN contribution to the total radio emission corresponds to the offset from the peak of the $\log \left(L_{1.4 \mathrm{GHz}} / \mathrm{SFR}_{\mathrm{IR}}\right)$ distribution at a given redshift. In the above relation, $L_{1.4 \mathrm{GHz}}$ is the radio luminosity and $\mathrm{SFR}_{\mathrm{IR}}$ is the star formation rate based on the integrated $8-1000 \mu \mathrm{m}$ SED, with any AGN component subtracted, using the Kennicutt (1998) relation and the Chabrier (2003) initial mass function. Galaxies below such a defined radio excess threshold might still host an AGN (visible in X-rays for example), but the radio emission originates mostly in star formation processes. The peak of the $L_{1.4 \mathrm{GHz}} / \mathrm{SFR}_{\mathrm{IR}}$ distribution shifts to higher values with increasing redshift, hence the need for a redshift-dependent description of the radio excess. Further evidence for the necessity of this redshift evolution arises from the analysis of the IR-radio correlation performed by Delhaize et al. (2017), where the authors find a decreasing trend in the IR-radio correlation parameter $q_{\mathrm{TIR}}$ with redshift (see also Ivison et al. 2010; Magnelli et al. 2015).

Radio LFs across cosmic time are usually described by a local LF evolved in luminosity, or density, or both (e.g., Condon 1984). Following N17 we can parametrize the redshift-dependent evolution with two parameters for density evolution $\left(\alpha_{D}, \beta_{D}\right)$ and two parameters for luminosity evolution $\left(\alpha_{L}, \beta_{L}\right)$ to obtain

$$
\Phi\left(L, z, \alpha_{L}, \beta_{L}, \alpha_{D}, \beta_{D}\right)=(1+z)^{\alpha_{D}+z \cdot \beta_{D}} \times \Phi_{0}\left[\frac{L}{(1+z)^{\alpha_{L}+z \cdot \beta_{L}}}\right],
$$

where $\Phi_{0}$ is the local LF. The linear form $\alpha+z \beta$ of the evolution parameter was chosen for its simplicity and its good fit to the data. Alternative multi-parameter descriptions also exist in the literature (see, e.g., Padovani et al. 2015; Gruppioni et al. 2013). The shape and the evolution of the LF depend on the galaxy population type. N17 used a power-law plus lognormal shape of the local LF for SF galaxies obtained as the best fit of the combined data from Condon et al. (2002), Best et al. (2005) and Mauch \& Sadler (2007)

$\Phi_{0}^{\mathrm{SF}}(L)=\Phi_{\star}\left(\frac{L}{L_{\star}}\right)^{1-\alpha} \exp \left[-\frac{1}{2 \sigma^{2}} \log ^{2}\left(1+\frac{L}{L_{\star}}\right)\right]$,

where $\Phi_{\star}=3.55 \times 10^{-3} \mathrm{Mpc}^{-3} \mathrm{dex}^{-1}, L_{\star}=1.85 \times 10^{21} \mathrm{~W} \mathrm{~Hz}^{-1}$, $\alpha=1.22$, and $\sigma=0.63$. They report the best-fit pure luminosity evolution (PLE) parameters $\alpha_{L}^{\mathrm{SF}}=3.16$ and $\beta_{L}^{\mathrm{SF}}=-0.32$, valid for $z<5.7$.

S17c used the double power-law shape reported in Mauch \& Sadler (2007) to describe the local AGN LF,

$\Phi_{0}^{\mathrm{AGN}}(L)=\frac{\Phi_{\star}}{\left(L_{\star} / L\right)^{\alpha}+\left(L_{\star} / L\right)^{\beta}}$,

where $\Phi_{\star}=\frac{1}{0.4} 10^{-5.5} \mathrm{Mpc}^{-3} \mathrm{dex}^{-1}, L_{\star}=10^{24.59} \mathrm{~W} \mathrm{~Hz}^{-1}$, $\alpha=-1.27$, and $\beta=-0.49$. They report the best-fit PLE parameters $\alpha_{L}^{\mathrm{AGN}}=2.88$ and $\beta_{L}^{\mathrm{AGN}}=-0.84$, valid for $z<5.5$. They also calculated the best-fit pure density evolution (PDE), which is consistent with their PLE. In Fig. 1 we show the luminosityevolved LFs (using Eq. (2) with $\alpha_{D}=0$ and $\beta_{D}=0$ ) of the SF galaxies and AGN (Eqs. (3) and (4)) as blue and red areas, respectively.

\subsection{Fitting the total luminosity function}

Following McAlpine et al. (2013), we fit the total radio LF with a combination of different LFs. Both PLE and PDE models are common in the literature (e.g., Condon 1984; Sadler et al. 2002; Gruppioni et al. 2013), but the true evolution might be a combination of both of these extremes (see, e.g., Yuan et al. 2016), with a possible luminosity-dependent evolution as well (see, e.g., Fotopoulou et al. 2016).

It was noted by N17 that the PDE of SF galaxies would push the densities to very high numbers, thus making them inconsistent with the observed cosmic star formation rate densities. This is a consequence of the fact that our data can constrain only the bright log-normal part of the SF LF. For AGN it was shown by S17c that the PDE and PLE models are similar, mostly because the shape of the LF does not deviate strongly from a simple power law at the observed luminosities. Considering the above reasoning while also trying to keep the parameter space degeneracy to a minimum, we decided to use only the PLE for our analysis.

We constructed a four-parameter redshift-dependent pure luminosity evolution model with two parameters for the SF and AGN populations. The total LF is defined by the following evolutionary model:

$$
\begin{aligned}
& \Phi\left(L, z, \alpha_{L}^{\mathrm{SF}}, \beta_{L}^{\mathrm{SF}}, \alpha_{L}^{\mathrm{AGN}}, \beta_{L}^{\mathrm{AGN}}\right)= \\
& \quad \Phi_{0}^{\mathrm{SF}}\left[\frac{L}{(1+z)^{\alpha_{L}^{\mathrm{SF}}+z \cdot \beta_{L}^{\mathrm{SF}}}}\right]+\Phi_{0}^{\mathrm{AGN}}\left[\frac{L}{\left.(1+z)^{\alpha_{L}^{\mathrm{AGN}}+z \cdot \beta_{L}^{\mathrm{AGN}}}\right],}\right.
\end{aligned}
$$

where $\Phi_{0}^{\mathrm{SF}}$ and $\Phi_{0}^{\mathrm{AGN}}$ are the local LFs for the two galaxy populations.

We used the Markov chain Monte Carlo (MCMC) algorithm, available in the Python package EMCEE (Foreman-Mackey et al. 2013), to perform a multi-variate fit to the data in Table A.1. Covariance maps of the four fitted parameters are shown in Fig. 2. This plot shows that the total fit shows a slight degeneracy between SF and AGN populations, but the intersection of AGN and SF LFs is well constrained by our data (see Fig. 1), and we therefore consider our fit robust despite this small degeneracy. For an individual population, a higher $\alpha$ parameter correlates strongly with a steeper (more negative) value of $\beta$. The redshift dependence of the total evolution parameter $(\alpha+z \times \beta)$ is necessary to describe the observations at all redshifts. The best-fit parameter values are listed in Table 1 as model 1, which is also shown in Fig. 1 (black line).

Since the shape of the LF is defined analytically, it extends beyond the observed data. The extrapolation toward the faint 


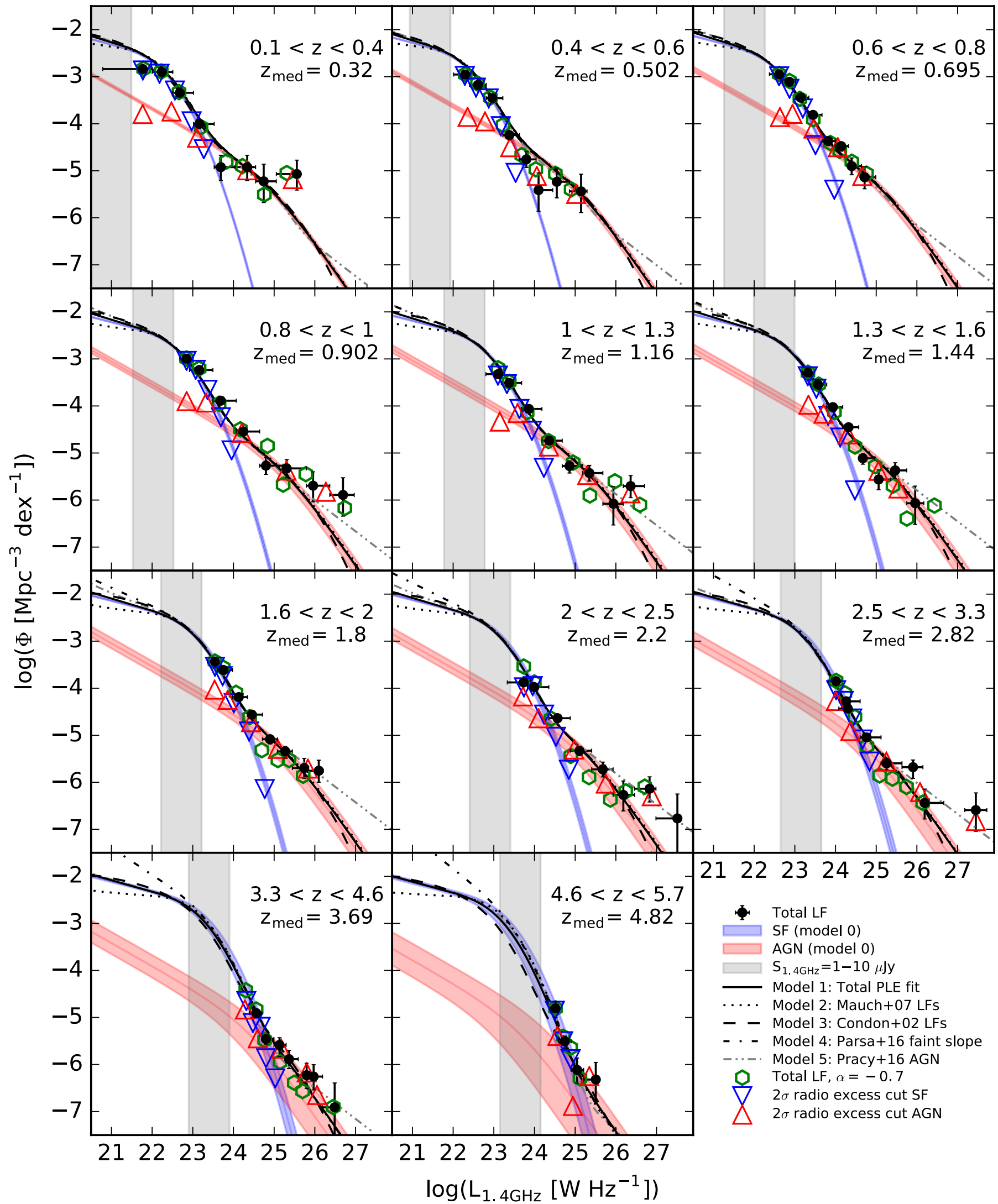

Fig. 1. Total radio LFs at different cosmic epochs. Black points show LFs derived using the $V_{\max }$ method (see Sect. 3.1). The blue and red shaded areas show the $\pm 3 \sigma$ ranges of the best-fit evolution for the individual SF and AGN populations, respectively (outlined in Sect. 3.2). The black line is the fit to the total radio LF, as explained in Sect. 3.3. Other models used are indicated in the legend. In all of these models we assume the local $(z \approx 0)$ LFs from the respective papers and fit for the PLE parameters as in Eq. (5). The only exception is model 4, where the shape of the faint slope of the SF LF changes with redshift. The vertical gray shaded area shows the luminosity decade that contributes to the radio source counts between 1 and $10 \mu \mathrm{Jy}$. 


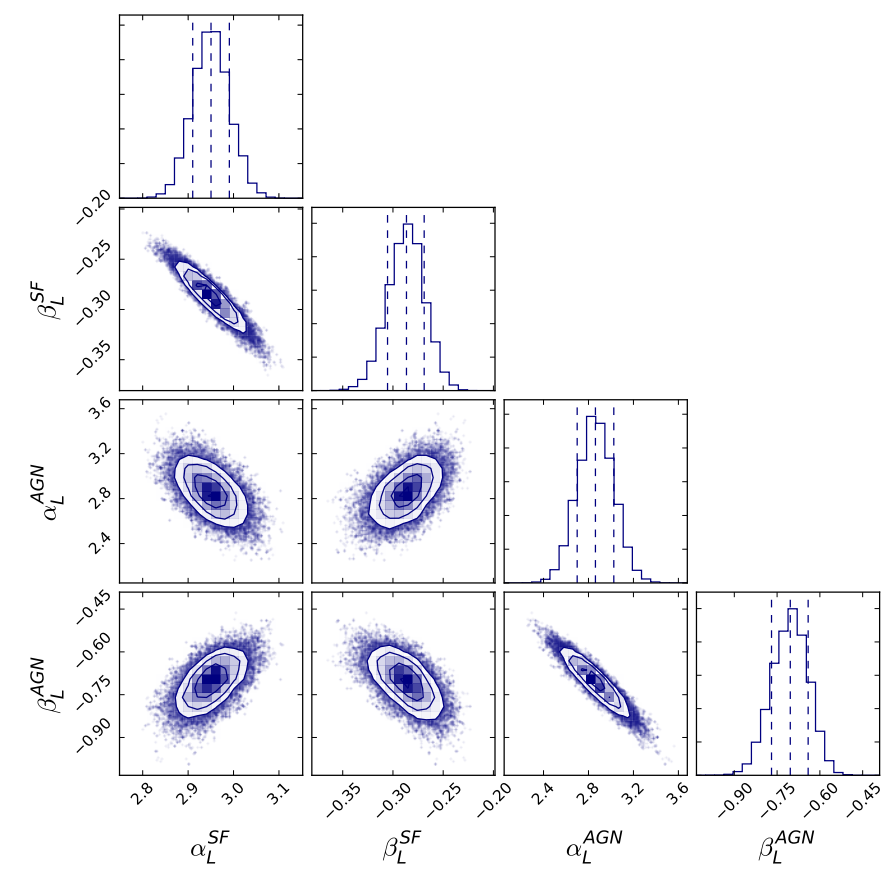

Fig. 2. Covariance maps of four fitting parameters used to describe the total radio LF evolution. Probability distribution histograms are also shown for each parameter with 16th, 50th, and 84th percentiles marked by vertical dashed lines.

end is particularly important (i.e., below $L_{1.4 \mathrm{GHz}}<10^{23} \mathrm{~W} \mathrm{~Hz}^{-1}$ at $z>1$ ) because the densities of galaxies are the highest in this range. A change in the faint-end slope of the LF can easily change the relative fractions of galaxy populations at faint flux densities. The only way to mitigate this extrapolation issue is to obtain deeper data. However, in the absence of such observations, we made use of different LFs. We performed additional MCMC fits using analytical SF and AGN LFs from Condon et al. (2002), where both LFs are hyperbolic in shape, Mauch \& Sadler (2007), and the redshift-dependent faint power-law slope for SF galaxies derived by Parsa et al. (2016). The best-fit evolution parameters are listed in Table 1, and the models are also shown in Fig. 1.

We additionally tried decomposing the AGN population into its two subpopulations (HEGs and LEGs) by adding a second AGN LF to Eq. (5). In this model we use the local LFs $(0.005<z<0.3)$ of LEGs and HEGs obtained by Pracy et al. (2016), which are based on the Large Area Radio Galaxy Evolution Spectroscopic Survey (LARGESS), and fit for the evolutionary parameters as before. We performed a six-parameter MCMC fit (two PLE parameters for each subpopulation) in an attempt to constrain the evolution of the total radio $\mathrm{LF} \Phi\left(L, z, \alpha_{L}^{\mathrm{SF}}, \beta_{L}^{\mathrm{SF}}, \alpha_{L}^{\mathrm{HEG}}, \beta_{L}^{\mathrm{HEG}}, \alpha_{L}^{\mathrm{LEG}}\right.$, and $\left.\beta_{L}^{\mathrm{LEG}}\right)$. The result is shown in Fig. 1 and is discussed in the next section.

\subsection{Discussing the fitted models and biases}

One advantage of performing a fit to the total radio LF is that the results are no longer sensitive to the galaxy classification method. However, it is important to assume a proper shape of the LFs for distinct galaxy populations in order to obtain a meaningful evolution fit. For this reason, the shape of the LF for a given population is fixed to the local LF, which is usually constrained across at least five orders of magnitudes in luminosities. The best-fit evolution parameters obtained from the MCMC on the total radio LF are consistent within the $3 \sigma$ errors with $\chi^{2}$ fits performed on individual populations (see shaded areas in Fig. 1). This good agreement between the results of different analyses implies that regardless of the specifics of the galaxy classification methods, if the local LFs for SF and AGN galaxies are well described with Eqs. (3) and (4), our PLE parameters are robust. However, the total radio LFs suggest slightly higher AGN densities than those obtained in S17c. Some redshift bins have some high-luminosity outliers, that is, data points that are above the best-fit LF. We now discuss potential biases that might cause these discrepancies.

\subsubsection{Spectral index bias}

The largest uncertainty in measuring the radio LF, especially evident at higher redshifts, is the imperfect knowledge of the radio SED. Under the assumption of a simple power law, an offset in spectral index of $\Delta \alpha=0.1$ would change the $1.4 \mathrm{GHz}$ restframe luminosity by 0.08 and 0.11 dex for $z=2$ and $z=5$, respectively. Previous studies have shown that there is a $\sim 0.4$ symmetric spread of spectral indices around the mean value (e.g., Kimball \& Ivezić 2008). A single power-law approximation might not hold if the radio SED consists of both thermal and nonthermal components (e.g., Tabatabaei et al. 2017). Additional deep radio data at both higher and lower frequencies are necessary to better understand this effect. Since we measured spectral indices using the $1.4 \mathrm{GHz}$ survey, which has a lower sensitivity, our measurements are biased toward steeper spectra. The usual assumption is that the spread in the spectral index will statistically cancel out for a large enough sample. This might not hold for the sparsely populated high-luminosity bins that at all redshifts contain a small number of galaxies (see Table A.1). Almost all of the brightest galaxies $\left(L_{1.4 \mathrm{GHz}}>10^{26} \mathrm{~W} \mathrm{~Hz}^{-1}\right)$ have a spectral index measurement, and $27 \%$ of them have a very steep spectrum $(\alpha<-1.5)$. When $\alpha=-0.7$ is assumed for all sources, the high-luminosity outliers are no longer present in the three redshift bins above $z>2$ (see Fig. 1). The total radio $\mathrm{LF}$ is also generally lower in the AGN regime, thus improving the agreement with the individual population fits. It is also worthwhile to mention that catastrophic failures of the photometric redshift estimates might also produce the observed outliers as photometric redshifts are used for $76 \%$ of these bright objects.

\subsubsection{Population classification bias}

The radio excess criterion from Eq. (1) was designed to select AGN with high purity; by reducing the threshold, more galaxy composites would be reclassified as AGN. To investigate this effect, we relaxed the radio excess selection criterion from $3 \sigma$ to $2 \sigma$ significance. This is achieved by setting the Eq. (1) parameters to $a=21.8$ and $b=0.011$. The estimated AGN contribution to the total emitted radio power in such a sample, measured from the offset from the peak of the $\log \left(L_{1.4 \mathrm{GHz}} / \mathrm{SFR}_{\mathrm{IR}}\right)$ distribution, is larger than $65 \%$. We have recalculated the radio LFs of individual populations selected in this manner (shown with triangles in Fig.1). We found that the newly derived LFs are still well described with the local LFs from Eqs. (3) and (4) evolved in luminosity. The AGN LF is systematically shifted to higher densities/luminosities, and the opposite is true for the SF LF. This change of the selection criterion mostly affects the faint observed end of the AGN LF and the bright end of the SF LF. These new fits fall within the $3 \sigma$ shaded areas in Fig. 1, strengthening our previous results, and the LFs of the 
two populations classified with the $2 \sigma$ selection cut are in better agreement with the fit performed on the total radio LF. If the selection cut were relaxed even further to just $1 \sigma$ radio excess (average AGN contribution larger than 40\%), our measured LFs would no longer be consistent with the local LFs. The number density of AGN at the faint end would be too high, while the bright end would remain unchanged. Therefore the $1 \sigma$ radio excess cut should probably not be used when dividing the radio emission into AGN or SF dominated.

Given the dichotomy of the AGN galaxy population discussed in Sect. 1, the AGN LF should be additionally decomposed into two separate LFs, each one evolving differently (e.g., Pracy et al. 2016). Wide area observations are necessary to obtain a statistically significant sample of the brightest and the rarest objects, and the COSMOS field is not best suited for such an analysis. Nevertheless, we attempted to fit our total radio LF with a three-population model. The AGN below $L_{1.4 \mathrm{GHz}}=10^{26} \mathrm{~W} \mathrm{~Hz}^{-1}$ observed locally are predominantly LEGs, while HEGs would be observed at higher luminosities (see Pracy et al. 2016). At higher redshifts the data are preferably fitted with the simple power-law used to describe the HERG population. Because of the large parameter degeneracy and insufficient number of sources, we do not report the obtained best-fit parameter values. The three-population model can explain the highest luminosity outliers, but it cannot be distinguished from possible spectral index systematics (as explained above). Given the limitation of the data, the two population model describes our data the best. Our model is best understood as a tracer of the radio emission origin (star formation or AGN), even though the dominant AGN host type, as classified from optical spectra, changes with redshift (see also Kimball et al. 2011). Our LFs can be further used to estimate, for example, the cosmic star formation rate density or the total AGN kinetic power density.

The SF population would also benefit from a further decomposition into differently evolving normal/disk galaxies, starbursts/mergers and galaxies with low level AGN activity (see, e.g., Hopkins et al. 2010), but a classification like this is beyond the scope of this paper and would introduce too many degenerate parameters into our models.

Completeness corrections used to calculate the $V_{\max }$ might also result in population-based biases. We assume that the completeness curve constructed in Smolčić et al. (2017a) equally applies to all radio sources. However, the authors found a strong dependence of the incompleteness on the intrinsic source size distribution. Specifically, if AGN were more compact and therefore easier to detect in a survey with a given surface brightness sensitivity, our AGN number densities might be overestimated. The intrinsic radio source size distributions for different classes of radio sources are still not constrained well enough to investigate this effect further and will be presented elsewhere (Bondi et al. in prep.).

\section{Radio number counts}

With several LF evolution models established in previous chapters, we now aim to calculate the radio number counts down to submicrojansky flux densities. This limit was chosen to encompass the limits of future deep radio surveys, and to enable comparison with the literature. The number of galaxies $\Delta N$ in a given spherical shell volume $\Delta V$ and a luminosity decade $\Delta \log L$ is given by the definition of the LF $\Phi$ as

$\Delta N=\Phi(L, z) \Delta V \Delta \log L$.

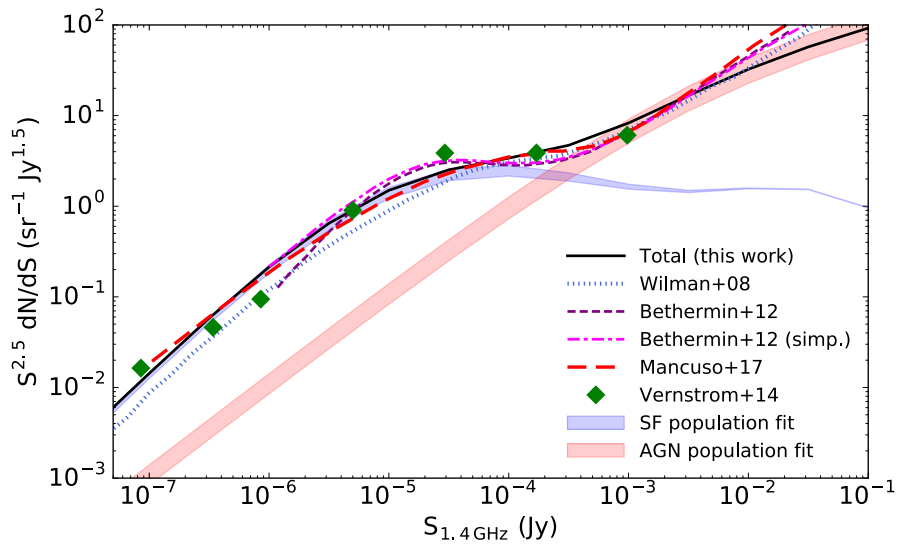

Fig. 3. Euclidean normalized radio source number counts drawn from LFs described in Sect. 3.2 compared to the literature values indicated in the legend. The shaded areas encompass the $3 \sigma$ errors from the $\chi^{2}$ fits performed on individual populations.

We compare our number counts with the results from semi-analytical models obtained by Wilman et al. (2008) and Béthermin et al. (2012) in Fig. 3. They are all in agreement at a $1.4 \mathrm{GHz}$ flux density of $100 \mu \mathrm{Jy}$. Our modeled number counts are systematically slightly higher at fainter flux densities (below $100 \mu \mathrm{Jy}$ at $1.4 \mathrm{GHz}$ ) when compared to the Wilman et al. (2008) model, an offset probably caused by the choice of the LF and its evolution. Below $5 \mu \mathrm{Jy}$ at $1.4 \mathrm{GHz}$ our number counts are increasingly higher than the Béthermin et al. (2012) model, reaching a factor two difference at $\sim 1 \mu \mathrm{Jy}$. On the other hand, the agreement with their simplified model is excellent in this range (see dash-dotted magenta line in Fig. 3). One of the refinements that the authors implemented in their simplified model in order to obtain their final result was the dust attenuation. This in practice flattens the faint end of the IR LF and probably causes the discrepancy below $5 \mu \mathrm{Jy}$. Because of the uncertainty of dust obscuration and its dependence on redshift, the dust-unbiased aspect of radio observations provides a valuable addition to galaxy evolution studies. Additionally, Béthermin et al. (2012) modeled only the X-ray selected AGN and ignored those associated with red sequence galaxies, which are easily identified in the radio band as AGN. We also compared our number counts with the work performed by Mancuso et al. (2017), who used the evolving cosmic SFR function obtained from the FIR, ultraviolet (UV), and $\mathrm{H} \alpha$ data to estimate radio (synchrotron and free-free emission) LFs. We find a good agreement with their work down to $0.1 \mu \mathrm{Jy}$.

An important result stems from the comparison of our counts with those obtained by Vernstrom et al. (2014). These investigators modeled the confusion amplitude distribution $P(D)$ from deep VLA $3 \mathrm{GHz}$ observations of the Lockman Hole. Their analysis uses the noise distribution of the radio map to constrain the underlying radio number counts down to $S_{3 \mathrm{GHz}} \sim 50 \mathrm{nJy}$. A great advantage of their approach is that it is not affected by incompleteness issues from direct counting and counterpart cross-correlations, and can be used to probe the radio sky properties below the nominal sensitivity limit for source detection. The excellent agreement between the results from their blind method and our counts suggests that the evolving LFs published in N17 and S17c provide plausible cosmic densities of galaxies, even though they rely on uncertain extrapolations toward the faint end. There is no need for a potential third population of radio sources (e.g., dwarf galaxies) at $0.1-1 \mu \mathrm{Jy}$ levels, and these LFs can reproduce the observed radio sky background. The 
Table 1. Best-fit PLE parameters obtained from the multivariate fitting to the total radio LF using two populations as given in Eq. (5).

\begin{tabular}{cccccc}
\hline \hline Model & Description & $\alpha_{L}^{\mathrm{SF}}$ & $\beta_{L}^{\mathrm{SF}}$ & $\alpha_{L}^{\mathrm{AGN}}$ & $\beta_{L}^{\mathrm{AGN}}$ \\
\hline 0 & Subpopulations* & $3.16 \pm 0.04$ & $-0.32 \pm 0.02$ & $2.88 \pm 0.17$ & $-0.84 \pm 0.07$ \\
1 & PLE fit to the total LF & $2.95 \pm 0.04$ & $-0.29 \pm 0.02$ & $2.86 \pm 0.16$ & $-0.70 \pm 0.06$ \\
2 & Mauch \& Sadler (2007) local LFs & $2.77 \pm 0.04$ & $-0.23 \pm 0.02$ & $3.05 \pm 0.15$ & $-0.76 \pm 0.06$ \\
3 & Condon et al. (2002) local LFs & $3.58 \pm 0.04$ & $-0.45 \pm 0.02$ & $2.46 \pm 0.16$ & $-0.60 \pm 0.06$ \\
4 & Parsa et al. (2016) faint slope & $3.04 \pm 0.04$ & $-0.19 \pm 0.02$ & $2.93 \pm 0.16$ & $-0.73 \pm 0.06$ \\
\hline
\end{tabular}

Notes. ${ }^{(*)}$ Fits on individual populations published in N17 and S17c. We note that reported errors on the fitted parameters are corrected (reduced) here to the listed $1 \sigma$ uncertainties.

largest discrepancy between the Vernstrom et al. (2014) results and ours is present in the $10-100 \mu \mathrm{Jy}$ range. Although the formal errors on the individual fits of Vernstrom et al. (2014) are small, the spread can be up to $30 \%$ depending on the area they analyze (their zones 1-3). In addition, they based their result on a field of 0.02 square degrees, which is 100 times smaller than the COSMOS field. Cosmic variance may therefore have a significant impact on their result (see Moster et al. 2011). This issue has previously been identified and tested by Smolčić et al. (2017a), since the flux densities above $10 \mu \mathrm{Jy}$ are also above the $5 \sigma$ sensitivity limit of the VLA-COSMOS $3 \mathrm{GHz}$ Large Survey (see their Fig. 18). It was shown that the sample variance obtained by splitting the COSMOS field into 100 smaller areas was enough to bring the two measurements into agreement.

Our number counts at the bright end exhibit a turnover at somewhat lower flux densities when compared to semi-analytical models (derived from abundant observations at $S_{1.4 \mathrm{GHz}}>1 \mathrm{mJy}$, see de Zotti et al. 2010). This is probably due to an overly simplified treatment of the brightest AGN. However, we emphasize that our sample is optimized to probe the faint radio sky and SF galaxies.

\subsection{The faint radio sky: what will the SKA see?}

We consider the number counts obtained from the MCMC fit described in Sect. 3.3 (model 1) as our main result and list them in Table 2. To quantify possible issues due to the faintend slope of the LF, we tried evolving different LFs available in the literature. All models are shown in Fig. 4. Overall, they are consistent among themselves, although with some expected variations below $1 \mu \mathrm{Jy}$.

By comparing the radio and the UV LFs, N17 noted that the evolved local radio LF might have a faint-end slope (below $L_{1.4 \mathrm{GHz}}<10^{22}$ ) that is too flat when compared to UV LFs at high redshift $(z>3)$. To obtain some insight into this effect, we have constructed a fourth model that uses a redshift-dependent faint-end slope, derived by Parsa et al. (2016), to describe UV LFs. This model is the same as our first model, with the exception that the faint slope parameter for SF galaxies ( $\alpha$ in Eq. (3)) now changes linearly with redshift as $\alpha=0.106 z+1.187$. As expected, the result of this LF choice is an increased density of faint SF galaxies, higher than either the Mancuso et al. (2017) or Vernstrom et al. (2014) estimates.

In Fig. 5 we show the fraction of each population as a function of flux density to better illustrate the effect of how relative abundances of SF galaxies and AGN change with telescope sensitivity. Several conclusions can be drawn from this plot. Above $1 \mathrm{mJy}$ at $1.4 \mathrm{GHz}$, the majority of radio emission is due to AGN, as is well known from past studies. At around
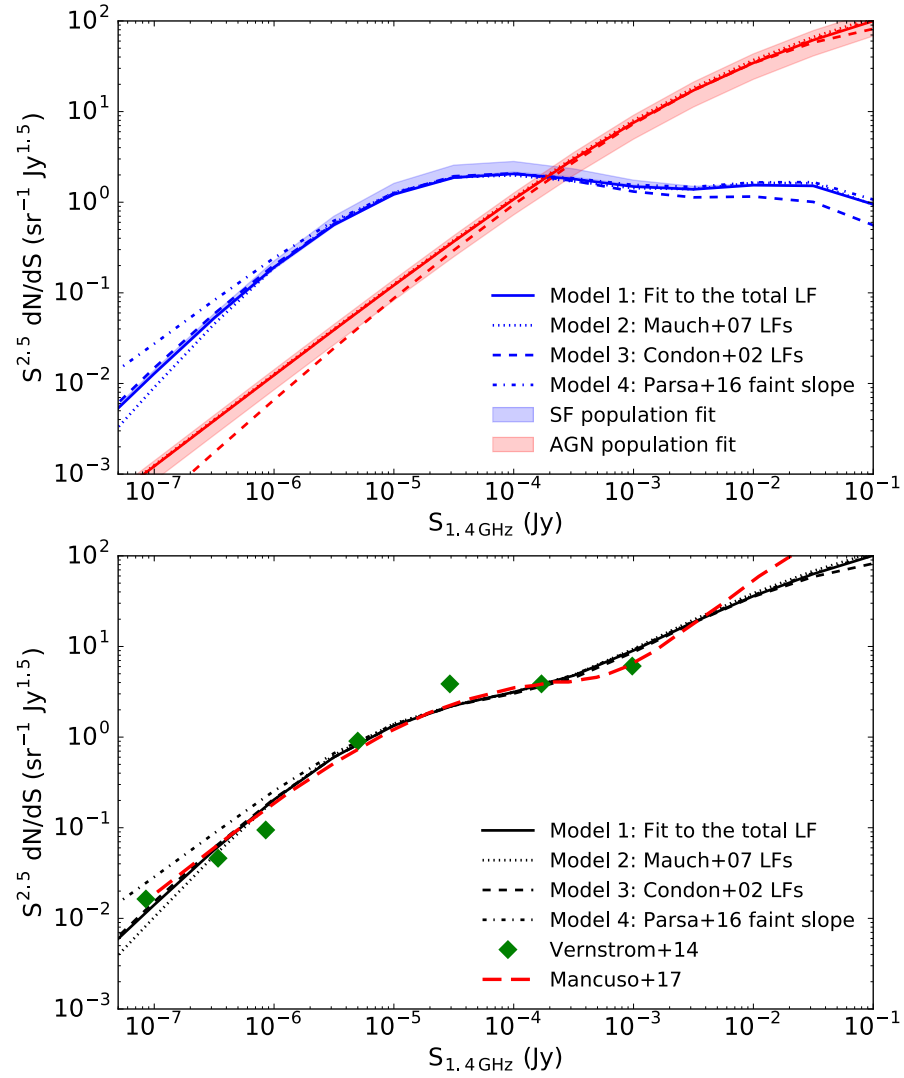

Fig. 4. Top panel: Euclidean normalized radio source number counts obtained by fitting the total radio LF using different evolving analytical LFs with the MCMC algorithm. Red lines (AGN) have the same line styles as blue lines (SF), as indicated in the legend. The shaded areas are equivalent to those in Fig. 3. Bottom panel: total number counts for different models obtained by adding together SF and AGN contributions.

$200 \mu \mathrm{Jy}$ at $1.4 \mathrm{GHz}$, both populations contribute equally to the observed counts, and at fainter flux densities, SF galaxies become the dominant population. The most important result is that between 0.1 and $10 \mu \mathrm{Jy}$, where the $5 \sigma$ sensitivity limits for future SKA surveys at $1.4 \mathrm{GHz}$ are located (see Prandoni $\&$ Seymour 2015), the relative fraction is roughly constant with around $90-95 \%$ of the radio emission originating from star formation. The implication of this result is that a simple flux density cut at $S_{1.4 \mathrm{GHz}}<10 \mu \mathrm{Jy}$ will select samples with less than $10 \%$ contamination by AGN radio emission, thus providing constraints on the radio emission origin.

We further analyzed our different models of evolving radio LFs and derived the redshift distribution of SF galaxies 
Table 2. Euclidean normalized differential radio number counts as a function of flux density at $1.4 \mathrm{GHz}$.

\begin{tabular}{cccc}
\hline \hline $\begin{array}{c}\log \left(S_{1.4 \mathrm{GHz}}\right) \\
{[\mathrm{Jy}]}\end{array}$ & $\begin{array}{c}\log \left(n^{\mathrm{SF}}\right) \\
{\left[\mathrm{sr}^{-1} \mathrm{Jy}^{1.5}\right]}\end{array}$ & $\begin{array}{c}\log \left(n^{\mathrm{AGN}}\right) \\
{\left[\mathrm{sr}^{-1} \mathrm{Jy}^{1.5}\right]}\end{array}$ & $\begin{array}{c}\log \left(n^{\text {Total }}\right) \\
{\left[\mathrm{sr}^{-1} \mathrm{Jy}^{1.5}\right]}\end{array}$ \\
\hline-7.5 & -2.52 & -3.42 & -2.47 \\
-7.0 & -1.89 & -2.91 & -1.85 \\
-6.5 & -1.28 & -2.41 & -1.25 \\
-6.0 & -0.717 & -1.91 & -0.690 \\
-5.5 & -0.250 & -1.41 & -0.221 \\
-5.0 & 0.0865 & -0.917 & 0.128 \\
-4.5 & 0.271 & -0.434 & 0.350 \\
-4.0 & 0.314 & 0.0330 & 0.497 \\
-3.5 & 0.256 & 0.474 & 0.680 \\
-3.0 & 0.173 & 0.878 & 0.956 \\
-2.5 & 0.142 & 1.23 & 1.27 \\
-2.0 & 0.188 & 1.54 & 1.56 \\
-1.5 & 0.180 & 1.79 & 1.80 \\
-1.0 & -0.0234 & 2.00 & 2.00 \\
\hline
\end{tabular}

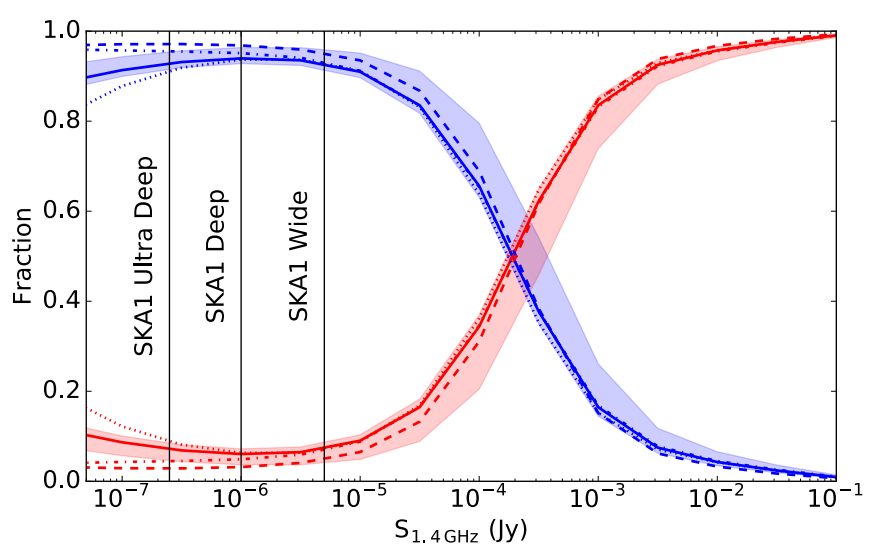

Fig. 5. Percentages of AGN (red) and SF galaxies (blue) as a function of $1.4 \mathrm{GHz}$ flux density. Colors and line styles are the same as in Fig. 4.

in different flux density ranges as presented in Fig. 6. Different models can yield similar number counts, but with different intrinsic redshift distributions. The VLA-COSMOS $3 \mathrm{GHz}$ Large Project survey has a median $5 \sigma$ sensitivity of $11.5 \mu \mathrm{Jy} \mathrm{beam}^{-1}$ and around $90 \%$ of all radio sources with optical/NIR counterparts fall inside the $1.4 \mathrm{GHz}$ flux density range of $10-100 \mu \mathrm{Jy}$. As can be seen from the figure, a large portion of our observed galaxies is distributed around a redshift of $z \sim 1$. Our models predict that only by probing the submicrojansky population $(0.1-1 \mu \mathrm{Jy})$ a significant shift of the source redshift distribution (peak at $z \sim 2$ ) can be obtained.

\subsection{Future surveys across radio bands}

The Euclidean normalized number counts $n$ at $1.4 \mathrm{GHz}$ reported in Table 2 can be converted into any other radio frequency $v$ under an assumption of a simple power-law radio SED with a spectral index $\alpha$ (usually $\alpha=-0.7$ ) taking $S_{v}=S_{1.4 \mathrm{GHz}} / f$ and $n_{v}=n_{1.4 \mathrm{GHz}} / f^{1.5}$, where $f=(1.4 / v[\mathrm{GHz}])^{\alpha}$ is the conversion factor, $n_{v}\left(S_{v}\right)$ are the number counts (flux densities) at the frequency $v$ one converts to. While this conversion is probably correct on average for most situations, it might be biased if the average radio SED of a galaxy population deviates

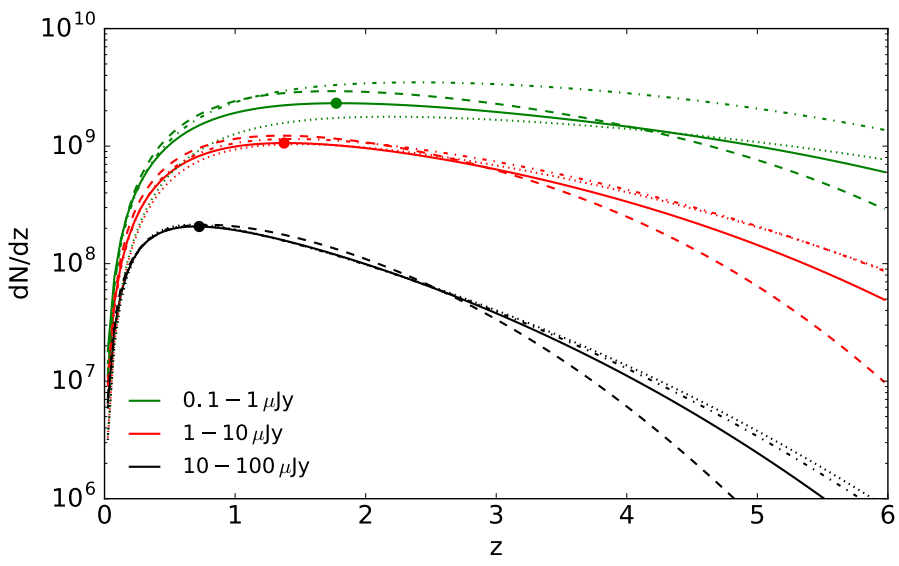

Fig. 6. Redshift distributions (differential numbers across the full sky, i.e., $4 \pi$ solid angle) of SF galaxies in different flux density decades based on evolving radio LFs at $1.4 \mathrm{GHz}$. Filled circles show the peaks of model 1. Different line styles describe models 1-4 as in Fig. 4.

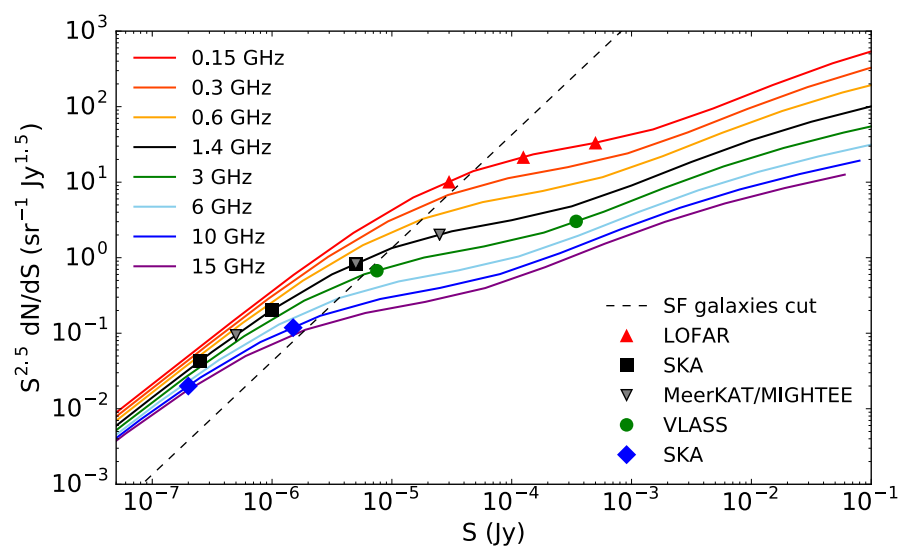

Fig. 7. Euclidean normalized radio number counts at different frequencies scaled from $1.4 \mathrm{GHz}$ with a simple power-law radio SED (see text for details). The dashed line marks the $S_{1.4 \mathrm{GHz}}=10 \mu \mathrm{Jy}$ cut, below which SF galaxies highly dominate the number counts. The filled symbols represent the $5 \sigma$ sensitivity limits for proposed multi-tiered (ultra-deep, deep, and wide) future radio surveys.

from a simple power law. For example, thermal free-free emission might provide a significant contribution to the total radio emission in SF galaxies at frequencies above $10 \mathrm{GHz}$. Below $150 \mathrm{MHz}$, synchrotron self-absorption might also affect the radio source counts. Additionally, radiative losses can impact the highfrequency counts of AGN. As deeper surveys across a wide radio bandwidth become available in the future, a comparison with this simple approach may be helpful to better quantify biases of radio SED assumptions.

We plot the number counts from our model 1 scaled to different frequencies in Fig. 7, where we also show the $5 \sigma$ sensitivities of several planned radio continuum surveys (see also Norris et al. 2013; Prandoni \& Seymour 2015). All of these surveys have multiple tiers stemming from the "wedding cake" observing approach: from wide and shallow observations spanning thousands of square degrees, to pencil-beam-deep and ultradeep observations. In the near future, observations such as the LOFAR $^{7}$ survey at $150 \mathrm{MHz}$ (see Morganti et al. 2010 and also Shimwell et al. 2017) or the $\operatorname{VLASS}^{8}$ at $3 \mathrm{GHz}$ will be able to

\footnotetext{
7 The LOw-Frequency ARray.

8 The Very Large Array Sky Survey https://science.nrao.edu/ science/surveys/vlass
} 
provide a solely radio-based selection of SF galaxies only at the sensitivity limits of their deep tier observations.

\section{Summary}

We used the VLA-COSMOS $3 \mathrm{GHz}$ Large Project radio data (Smolčić et al. 2017a) to construct the total radio luminosity functions extending out to redshift $z<5.7$ from a sample of 7826 radio sources within an area of $1.77 \mathrm{deg}^{2}$ with robust optical/NIR counterparts. We fitted the total radio LF with pure luminosity evolution models that depend on redshift. They rely on the sum of differently evolving SF and AGN LFs. We tested LFs of star-forming and AGN galaxies published in Novak et al. (2017) and Smolčić et al. (2017c) and found them to be consistent with the MCMC fits performed in this paper. We have tested our radio excess selection criteria against the total radio $\mathrm{LF}$, and have found that both $3 \sigma$ and $2 \sigma$ cuts are consistent with our previously published LFs. The total radio LF is in better agreement with the $2 \sigma$ cut. However, it is difficult to distinguish selection effects from potential spectral index systematics. We calculated radio number counts from our evolution models down to submicrojansky levels. We found that our LFs can well reproduce the number counts obtained from a blind probability of deflection $P(D)$ analysis (Vernstrom et al. 2014), implying that our extrapolations toward the faint end of the LFs are plausible even though they are not constrained directly by the data. Finally, we showed that planned surveys with the SKA will almost exclusively probe the star-forming galaxy population with a peak in the redshift distribution at $z \sim 2$. Our results suggest that the radio sky between 0.1 and $10 \mu \mathrm{Jy}$ at $1.4 \mathrm{GHz}$ is dominated by star formation processes with a maximum of $\sim 10 \%$ contamination from the radio AGN population. Future radio surveys will be able to provide an additional simple diagnostic for classification of galaxies across large areas based solely on radio flux densities.

Acknowledgements. We thank the anonymous referee for helpful suggestions that improved the quality of the paper. This research was funded by the European Unions Seventh Frame-work program under grant agreement 337595 (ERC Starting Grant, CoSMass).

\section{References}

Baloković, M., Smolčić, V., Ivezić, Ž., et al. 2012, ApJ, 759, 30

Best, P. N., \& Heckman, T. M. 2012, MNRAS, 421, 1569

Best, P. N., Kauffmann, G., Heckman, T. M., \& Ivezić, Ž. 2005, MNRAS, 362, 9

Béthermin, M., Daddi, E., Magdis, G., et al. 2012, ApJ, 757, L23

Bonzini, M., Padovani, P., Mainieri, V., et al. 2013, MNRAS, 436, 3759

Capak, P., Aussel, H., Ajiki, M., et al. 2007, ApJS, 172, 99

Chabrier, G. 2003, PASP, 115, 763

Clemens, M. S., Vega, O., Bressan, A., et al. 2008, A\&A, 477, 95

Condon, J. J. 1984, ApJ, 287, 461

Condon, J. J. 1989, ApJ, 338, 13

Condon, J. J. 1992, ARA\&A, 30, 575

Condon, J. J., Cotton, W. D., \& Broderick, J. J. 2002, AJ, 124, 675

Condon, J. J., Cotton, W. D., Fomalont, E. B., et al. 2012, ApJ, 758, 23

de Zotti, G., Massardi, M., Negrello, M., \& Wall, J. 2010, A\&ARv, 18, 1

Delhaize, J., Smolčić, V., Delvecchio, I., et al. 2017, A\&A, 602, A4

Delvecchio, I., Smolčić, V., Zamorani, G., et al. 2017, A\&A, 602, A3

Evans, D. A., Worrall, D. M., Hardcastle, M. J., Kraft, R. P., \& Birkinshaw, M. 2006, ApJ, 642, 96

Fanidakis, N., Baugh, C. M., Benson, A. J., et al. 2011, MNRAS, 410, 53

Fixsen, D. J., Kogut, A., Levin, S., et al. 2011, ApJ, 734, 5

Foreman-Mackey, D., Hogg, D. W., Lang, D., \& Goodman, J. 2013, PASP, 125 306
Fotopoulou, S., Buchner, J., Georgantopoulos, I., et al. 2016, A\&A, 587, A142 Franzen, T. M. O., Sadler, E. M., Chhetri, R., et al. 2014, MNRAS, 439, 1212 Gendre, M. A., Best, P. N., Wall, J. V., \& Ker, L. M. 2013, MNRAS, 430, 3086 Gruppioni, C., Pozzi, F., Rodighiero, G., et al. 2013, MNRAS, 432, 23 Hale, C. L., Jarvis, M. J., Delvecchio, I., et al. 2018, MNRAS, 474, 4133 Hales, C. A., Murphy, T., Curran, J. R., et al. 2012, MNRAS, 425, 979 Hales, C. A., Norris, R. P., Gaensler, B. M., et al. 2014, MNRAS, 441, 2555 Hardcastle, M. J., Evans, D. A., \& Croston, J. H. 2006, MNRAS, 370, 1893 Hardcastle, M. J., Ching, J. H. Y., Virdee, J. S., et al. 2013, MNRAS, 429, 2407

Heckman, T. M., \& Best, P. N. 2014, ARA\&A, 52, 589

Heywood, I., Jarvis, M. J., \& Condon, J. J. 2013, MNRAS, 432, 2625

Hopkins, A. M., Afonso, J., Chan, B., et al. 2003, AJ, 125, 465

Hopkins, P. F., Younger, J. D., Hayward, C. C., Narayanan, D., \& Hernquist, L. 2010, MNRAS, 402, 1693

Ivison, R. J., Alexander, D. M., Biggs, A. D., et al. 2010, MNRAS, 402, 245

Kennicutt, Jr. R. C. 1998, ARA\&A, 36, 189

Kimball, A. E., \& Ivezić, Ž. 2008, AJ, 136, 684

Kimball, A. E., Kellermann, K. I., Condon, J. J., Ivezić, Ž., \& Perley, R. A. 2011, ApJ, 739, L29

Laigle, C., McCracken, H. J., Ilbert, O., et al. 2016, ApJS, 224, 24

Laing, R. A., Jenkins, C. R., Wall, J. V., \& Unger, S. W. 1994, in The Physics of Active Galaxies, eds. G. V. Bicknell, M. A. Dopita, \& P. J. Quinn, ASP Conf. Ser., 54, 201

Longair, M. S. 1966, MNRAS, 133, 421

Magnelli, B., Ivison, R. J., Lutz, D., et al. 2015, A\&A, 573, A45

Mancuso, C., Lapi, A., Prandoni, I., et al. 2017, ApJ, 842, 95

Mauch, T., \& Sadler, E. M. 2007, MNRAS, 375, 931

McAlpine, K., Jarvis, M. J., \& Bonfield, D. G. 2013, MNRAS, 436, 1084

Merloni, A., \& Heinz, S. 2008, MNRAS, 388, 1011

Mitchell-Wynne, K., Santos, M. G., Afonso, J., \& Jarvis, M. J. 2014, MNRAS, 437, 2270

Morganti, R., Rottgering, H., Snellen, I., et al. 2010, ArXiv e-prints [arXiv: 1001.2384$]$

Morić, I., Smolčić, V., Kimball, A., et al. 2010, ApJ, 724, 779

Moster, B. P., Somerville, R. S., Newman, J. A., \& Rix, H.-W. 2011, ApJ, 731 113

Norris, R. P., Afonso, J., Bacon, D., et al. 2013, PASA, 30, 20

Novak, M., Smolčić, V., Delhaize, J., et al. 2017, A\&A, 602, A5

Owen, F. N., \& Morrison, G. E. 2008, AJ, 136, 1889

Padovani, P. 2016, A\&ARv, 24, 13

Padovani, P., Mainieri, V., Tozzi, P., et al. 2009, ApJ, 694, 235

Padovani, P., Bonzini, M., Kellermann, K. I., et al. 2015, MNRAS, 452, 1263

Padovani, P., Alexander, D. M., Assef, R. J., et al. 2017, A\&ARv, 25, 2

Parsa, S., Dunlop, J. S., McLure, R. J., \& Mortlock, A. 2016, MNRAS, 456, 3194

Pracy, M. B., Ching, J. H. Y., Sadler, E. M., et al. 2016, MNRAS, 460, 2

Prandoni, I., \& Seymour, N. 2015, AASKA14, 67

Rau, U., \& Cornwell, T. J. 2011, A\&A, 532, A71

Rowan-Robinson, M., Benn, C. R., Lawrence, A., McMahon, R. G., \& Broadhurst, T. J. 1993, MNRAS, 263, 123

Sadler, E. M., Jenkins, C. R., \& Kotanyi, C. G. 1989, MNRAS, 240, 591

Sadler, E. M., Jackson, C. A., Cannon, R. D., et al. 2002, MNRAS, 329, 227

Saunders, W., Rowan-Robinson, M., Lawrence, A., et al. 1990, MNRAS, 242, 318

Scheuer, P. A. G. 1957, Proc. Camb. Philos. Soc., 53, 764

Schinnerer, E., Sargent, M. T., Bondi, M., et al. 2010, ApJS, 188, 384

Schmidt, M. 1968, ApJ, 151, 393

Seymour, N., McHardy, I. M., \& Gunn, K. F. 2004, MNRAS, 352, 131

Shimwell, T. W., Röttgering, H. J. A., Best, P. N., et al. 2017, A\&A, 598, A104

Smolčić, V. 2009, ApJ, 699, L43

Smolčić, V. 2015, The Many Facets of Extragalactic Radio Surveys: Towards New Scientific Challenges, 22

Smolčić, V., Novak, M., Bondi, M., et al. 2017a, A\&A, 602, A1

Smolčić, V., Delvecchio, I., Zamorani, G., et al. 2017b, A\&A, 602, A2

Smolčić, V., Novak, M., Delvecchio, I., et al. 2017c, A\&A, 602, A6

Tabatabaei, F. S., Schinnerer, E., Krause, M., et al. 2017, ApJ, 836, 185

Vernstrom, T., Scott, D., \& Wall, J. V. 2011, MNRAS, 415, 3641

Vernstrom, T., Scott, D., Wall, J. V., et al. 2014, MNRAS, 440, 2791

Vernstrom, T., Scott, D., Wall, J. V., et al. 2016a, MNRAS, 462, 2934

Vernstrom, T., Scott, D., Wall, J. V., et al. 2016b, MNRAS, 461, 2879

Wilman, R. J., Miller, L., Jarvis, M. J., et al. 2008, MNRAS, 388, 1335

Yuan, Z., Wang, J., Zhou, M., \& Mao, J. 2016, ApJ, 820, 65

Zwart, J., Wall, J., Karim, A., et al. 2015a, AASKA14, 172

Zwart, J. T. L., Santos, M., \& Jarvis, M. J. 2015b, MNRAS, 453, 1740 
Appendix A: Tables

Table A.1. LFs of the total radio-selected sample obtained with the $V_{\max }$ method.

\begin{tabular}{|c|c|c|c|c|c|c|c|c|}
\hline $\begin{array}{c}\log L_{1.4 \mathrm{GHz}} \\
{\left[\mathrm{W} \mathrm{Hz}{ }^{-1}\right]}\end{array}$ & $\begin{array}{c}\log \Phi \\
{\left[\mathrm{Mpc}^{-3} \mathrm{dex}^{-1}\right]}\end{array}$ & $N$ & $\begin{array}{c}\log L_{1.4 \mathrm{GHz}} \\
{\left[\mathrm{W} \mathrm{Hz}{ }^{-1}\right]}\end{array}$ & $\begin{array}{c}\log \Phi \\
{\left[\mathrm{Mpc}^{-3} \mathrm{dex}^{-1}\right]}\end{array}$ & $N$ & $\begin{array}{c}\log L_{1.4 \mathrm{GHz}} \\
{\left[\mathrm{W} \mathrm{Hz}{ }^{-1}\right]}\end{array}$ & $\begin{array}{c}\log \Phi \\
{\left[\mathrm{Mpc}^{-3} \mathrm{dex}^{-1}\right]}\end{array}$ & $N$ \\
\hline$z=0.1-0.4$ & & & $z=1.0-1.3$ & & & $z=2.5-3.3$ & & \\
\hline $21.77_{-0.99}^{+0.23}$ & $-2.84_{-0.068}^{+0.080}$ & 202 & $23.10_{-0.30}^{+0.080}$ & $-3.32_{-0.041}^{+0.045}$ & 228 & $24.01_{-0.20}^{+0.076}$ & $-3.85_{-0.043}^{+0.048}$ & 172 \\
\hline $22.24_{-0.24}^{+0.99}$ & $\begin{array}{r}-2.90_{-0.023}^{+0.008} \\
-0.024\end{array}$ & 352 & $23.38_{-0.19}^{+0.30}$ & $\begin{array}{r}-3.51_{-0.019}^{-0.041} \\
-3.019\end{array}$ & 542 & $24.26_{-0.17}^{-0.20}$ & $-4.28_{-0.024}^{+0.043}$ & 327 \\
\hline $22.68_{-0.17}^{+0.24}$ & $\begin{array}{r}-3.34_{-0.035}^{+0.023} \\
-0.03\end{array}$ & 145 & $23.86_{-0.18}^{-0.19}$ & $-4.06_{-0.031}^{+0.019}$ & 190 & $24.76_{-0.16}^{+0.36}$ & $\begin{array}{r}-0.024 \\
-5.05_{-0.051}^{+0.058}\end{array}$ & 67 \\
\hline $23.16_{-0.14}^{+0.17}$ & $\begin{array}{r}-00^{-0.035} \\
-4.0083 \\
-0.070\end{array}$ & 33 & $24.36_{-0.18}^{-0.18}$ & $\begin{array}{r}-4.74_{-0.062}^{+0.031} \\
-0.072\end{array}$ & 43 & $25.26_{-0.14}^{-0.10}$ & $\begin{array}{l}-5.60_{-0.10}^{+0.13} \\
-0.01\end{array}$ & 17 \\
\hline $23.69_{-0.16}^{+0.14}$ & $-4.92_{-0.25}^{+0.28}$ & 4 & $24.86_{-0.18}^{+0.18}$ & $\begin{array}{r}-0.062 \\
-5.28_{-0.11}^{+0.15}\end{array}$ & 12 & $25.91_{-0.27}^{+0.24}$ & $-5.68_{-0.15}^{+0.24}$ & 12 \\
\hline $24.34_{-0.21}^{+0.10}$ & $-4.92_{-0.25}^{+0.25}$ & 4 & $25.35_{-0.17}^{+0.18}$ & $-5.43_{-0.16}^{+0.11}$ & 9 & $26.19_{-0.042}^{+0.47}$ & $-6.44_{-0.30}^{+0.34}$ & 3 \\
\hline $24.74_{-0.20}^{+0.30}$ & $\begin{array}{r}-5.22_{-0.37}^{-0.45}\end{array}$ & 2 & $25.94_{-0.26}^{+0.11}$ & $\begin{array}{r}-6.08_{-0.37}^{+0.40} \\
\text { +0.45 }\end{array}$ & 2 & $27.45_{-0.27}^{+0.027}$ & $-6.59_{-0.37}^{+0.45}$ & 2 \\
\hline $25.56_{-0.50}^{+0.030}$ & $\begin{array}{r}-0.37 \\
-5.07_{-0.30}^{+0.34} \\
\end{array}$ & 3 & $26.36_{-0.18}^{+0.30}$ & $\begin{array}{r}-5.70_{-0.22}^{+0.31} \\
\end{array}$ & 5 & & & \\
\hline$z=0.4-0.6$ & & & $z=1.3-1.6$ & & & $z=3.3-4.6$ & & \\
\hline $22.30_{-0.29}^{+0.11}$ & $-2.95_{-0.043}^{+0.048}$ & 151 & $23.32_{-0.14}^{+0.068}$ & $-3.29_{-0.037}^{+0.040}$ & 173 & $24.30_{-0.22}^{+0.097}$ & $-4.43_{-0.070}^{+0.084}$ & 75 \\
\hline $22.61_{-0.21}^{-0.29}$ & $\begin{array}{r}-0.043 \\
-3.17_{-0.026}^{+0.027}\end{array}$ & 271 & $23.57_{-0.18}^{+0.22}$ & $-3.54_{-0.021}^{+0.022}$ & 432 & $24.56_{-0.16}^{-0.22}$ & $\begin{array}{r}-0.050 \\
-4.91_{-0.051}^{+0.058}\end{array}$ & 66 \\
\hline $22.96_{-0.15}^{+0.26}$ & $\begin{array}{r}-0.026 \\
-3.46_{-0.033}^{+0.036}\end{array}$ & 165 & $23.94_{-0.15}^{-0.18}$ & $\begin{array}{r}-0.021 \\
-4.02_{-0.031}^{+0.033}\end{array}$ & 188 & $24.80_{-0.10}^{+0.20}$ & $-5.46_{-0.087}^{+0.11}$ & 21 \\
\hline $23.38_{-0.16}^{+0.15}$ & $\begin{array}{r}-0.033 \\
-4.24_{-0.074}^{+0.089}\end{array}$ & 29 & $24.32_{-0.14}^{+0.15}$ & $\begin{array}{r}-0.031 \\
-4.45_{-0.049}^{+0.055}\end{array}$ & 72 & $25.13_{-0.14}^{+0.16}$ & $-5.58_{-0.16}^{+0.24}$ & 11 \\
\hline $23.80_{-0.17}^{+0.16}$ & $\begin{array}{r}-0.074 \\
-4.76_{-0.16}^{+0.17}\end{array}$ & 9 & $24.67_{-0.10}^{+0.14}$ & $\begin{array}{c}-0.049 \\
-5.11_{-0.098}^{+0.13}\end{array}$ & 16 & $25.37_{-0.072}^{-0.14}$ & $\begin{array}{r}-0.16 \\
-5.89_{-0.19}^{+0.20}\end{array}$ & 7 \\
\hline $24.10_{-0.060}^{+0.35}$ & $\begin{array}{r}-0.16 \\
-5.41_{-0.37}^{+0.45}\end{array}$ & 2 & $25.06_{-0.095}^{-0.10}$ & $\begin{array}{r}-0.098 \\
-5.56_{-0.20}^{+0.22}\end{array}$ & 6 & $25.80_{-0.21}^{+0.092}$ & $\begin{array}{r}-0.19 \\
-6.23_{-0.25}^{+0.28}\end{array}$ & 4 \\
\hline $24.55_{-0.099}^{+0.000}$ & $-5.23_{-0.30}^{+0.31}$ & 3 & $25.47_{-0.11}^{+0.28}$ & $\begin{array}{r}-0.20 \\
-5.37^{+0.17}\end{array}$ & 9 & $25.97_{-0.080}^{+0.21}$ & $\begin{array}{r}-0.25 \\
-6.26_{-0.25}^{+0.28}\end{array}$ & 4 \\
\hline $25.14_{-0.29}^{+0.15}$ & $-5.44_{-0.37}^{-0.30}$ & 2 & $25.96_{-0.21}^{+0.21}$ & $\begin{array}{r}-0.16 \\
-6.07_{-0.37}^{+0.45} \\
\end{array}$ & 2 & $26.49_{-0.30}^{+0.026}$ & $\begin{array}{r}-0.25 \\
-6.91_{-0.52}^{+0.76} \\
\end{array}$ & 1 \\
\hline$z=0.6-0.8$ & & & $z=1.6-2.0$ & & & $z=4.6-5.7$ & & \\
\hline $22.61_{-0.24}^{+0.080}$ & $-2.95_{-0.054}^{+0.062}$ & 190 & $23.54_{-0.19}^{+0.067}$ & $-3.43_{-0.054}^{+0.061}$ & 192 & $24.52_{-0.13}^{+0.077}$ & $-4.81_{-0.14}^{+0.20}$ & 14 \\
\hline $22.86_{-0.17}^{-0.15}$ & $\begin{array}{r}-0.011_{-0.023}^{+0.024} \\
-3.023\end{array}$ & 354 & $23.75_{-0.14}^{+0.19}$ & $\begin{array}{r}-3.61_{-0.019}^{+0.034} \\
-0.020\end{array}$ & 494 & $24.75_{-0.15}^{+0.13}$ & $\begin{array}{r}-5.51_{-0.11}^{+0.14} \\
+0.15\end{array}$ & 12 \\
\hline $23.14_{-0.12}^{+0.17}$ & $\begin{array}{r}-0.023 \\
-3.44_{-0.030}^{+0.032}\end{array}$ & 205 & $24.13_{-0.15}^{+0.14}$ & $\begin{array}{r}-4.19_{-0.032}^{+0.019} \\
-0.015\end{array}$ & 173 & $25.05_{-0.15}^{+0.16}$ & $\begin{array}{r}-6.12_{-0.25}^{+0.11} \\
-0.28\end{array}$ & 4 \\
\hline $23.45_{-0.10}^{+0.12}$ & $\begin{array}{r}-0.030 \\
-3.81_{-0.043}^{+0.048}\end{array}$ & 92 & $24.45_{-0.11}^{+0.13}$ & $\begin{array}{r}-4.56_{-0.049}^{-0.032} \\
-0.045\end{array}$ & 73 & $25.51_{-0.30}^{+0.035}$ & $\begin{array}{r}-6.32_{-0.37}^{-0.25} \\
-0.45\end{array}$ & 2 \\
\hline $23.82_{-0.15}^{-0.10}$ & $\begin{array}{r}-4.37_{-0.079}^{-0.043} \\
-0.096\end{array}$ & 26 & $24.90_{-0.19}^{+0.11}$ & $\begin{array}{r}-0.049 \\
-5.09_{-0.081}^{+0.099}\end{array}$ & 24 & & & \\
\hline $24.14_{-0.15}^{-0.15}$ & $-4.48_{-0.086}^{+0.11}$ & 21 & $25.27_{-0.19}^{-0.19}$ & $-5.34_{-0.11}^{+0.081}$ & 13 & & & \\
\hline $24.40_{-0.080}^{+0.15}$ & $\begin{array}{l}-0.00_{-0.18}^{+0.086} \\
-4.18\end{array}$ & 8 & $25.74_{-0.29}^{+0.075}$ & $\begin{array}{r}-5.70_{-0.20}^{-0.11} \\
-0.22\end{array}$ & 6 & & & \\
\hline $24.71_{-0.074}^{+0.28}$ & $-5.14_{-0.22}^{+0.25}$ & 5 & $26.10_{-0.28}^{+0.11}$ & $-5.75_{-0.22}^{+0.25}$ & 5 & & & \\
\hline$z=0.8-1.0$ & & & $z=2.0-2.5$ & & & & & \\
\hline $22.85_{-0.16}^{+0.072}$ & $\begin{array}{l}2.99_{-0.038}^{+0.042} \\
\end{array}$ & 184 & $23.73_{-0.40}^{+0.093}$ & $-3.88_{-0.040}^{+0.044}$ & 168 & & & \\
\hline $23.16_{-0.24}^{+0.33}$ & $\begin{array}{r}-0.038 \\
-3.24_{-0.017}^{+0.018}\end{array}$ & 647 & $23.99_{-0.17}^{+0.36}$ & $\begin{array}{r}-0.040 \\
-3.98_{-0.021}^{+0.022}\end{array}$ & 410 & & & \\
\hline $23.69_{-0.19}^{+0.34}$ & $-3.89_{-0.032}^{+0.031}$ & 177 & $24.57_{-0.21}^{+0.31}$ & $-4.64_{-0.040}^{+0.041}$ & 112 & & & \\
\hline $24.24_{-0.17}^{+0.19}$ & $\begin{array}{r}-0.032 \\
-4.54_{-0.062}^{+0.073}\end{array}$ & 42 & $25.10_{-0.23}^{+0.30}$ & $\begin{array}{c}-0.040 \\
-5.33_{-0.085}^{+0.11}\end{array}$ & 23 & & & \\
\hline $24.80_{-0.15}^{+0.42}$ & $-5.27_{-0.17}^{+0.18}$ & 8 & $25.68_{-0.27}^{+0.25}$ & $-5.73_{-0.15}^{+0.16}$ & 10 & & & \\
\hline $25.31_{-0.090}^{+0.48}$ & $-5.33_{-0.19}^{+0.20}$ & 7 & $26.18_{-0.25}^{+0.28}$ & $-6.27_{-0.30}^{+0.34}$ & 3 & & & \\
\hline $25.96_{-0.16}^{+0.040}$ & $-5.69_{-0.30}^{+0.34}$ & 3 & $26.83_{-0.37}^{+0.16}$ & $-6.14_{-0.25}^{+0.28}$ & 4 & & & \\
\hline $26.69_{-0.33}^{+0.10}$ & $-5.89_{-0.37}^{+0.45}$ & 2 & $27.51_{-0.53}^{+0.028}$ & $-6.77_{-0.52}^{+0.76}$ & 1 & & & \\
\hline
\end{tabular}

